\title{
LA INNOVACIÓN A TRAVÉS DE ENTORNOS VIRTUALES DE ENSEÑANZA Y APRENDIZAJE
}

\author{
(INNOVATION USING VIRTUAL ENVIRONMENTS FOR TEACHING AND LEARNING)
}

David Carabantes Alarcón

Amparo Carrasco Pradas

Joaquim Daniel Alves Pais

Universidad Complutense de Madrid (España)

\section{RESUMEN}

Este artículo analiza la utilización de espacios virtuales de enseñanza y aprendizaje para el desarrollo de innovación en docencia, investigación y gestión. La Universidad se beneficia del uso de entornos virtuales, existiendo una estrategia general para el diseño y desarrollo de prácticas efectivas, asistiendo a un importante cambio en las funciones y la relación entre profesor y estudiante. Las Escuelas Universitarias de Estudios Empresariales y de Enfermería, Fisioterapia y Podología de la Universidad Complutense de Madrid (España), han realizado distintas propuestas que muestran las ventajas de la implantación de espacios virtuales en la educación superior para alcanzar la autonomía y la independencia del alumno en su propio proceso de aprendizaje.

\begin{abstract}
This paper analyses the use of virtual environments for teaching and learning to innovate on education, investigation and management. University is benefiting from the use of virtual environments, a general strategy is proposed for the design and development of effective practices, and we are living an important change in the roles and the relation between of professor and student. The School of Business Studies and the School of Nursing, Physiotherapy and Podiatry of the Universidad Complutense de Madrid (Spain), have made different proposals that show the advantages of the implantation of virtual spaces on higher education to achieve the autonomy and independence of the student on his own learning process.
\end{abstract}




\section{INTRODUCCIÓN}

El término innovación ha adquirido una gran importancia en la actualidad, según Rivas (2000, p. 20) "innovación es la incorporación de algo nuevo dentro de una realidad existente, en cuya virtud ésta resulta modificada", idea que está en la línea con la de Cebrián (2003, p. 21), "definimos innovación asociándola en un primer momento con el concepto de cambio, porque ambos son dos conceptos muy relacionados, tanto que no entenderíamos uno sin el otro".

En el campo formativo existen diferentes enfoques, para Carbonell (2002, p. 11) "la innovación educativa, utilizada como sinónimo de renovación pedagógica, es un concepto complejo y polisémico -como lo son los de calidad educativa o libertad de enseñanza- que se presta a múltiples lecturas e interpretaciones. Aquí lo entendemos como un conjunto de ideas, proceso y estrategias, más o menos sistematizados, mediante las cuales se trata de introducir y provocar cambios en las prácticas educativas vigentes".

La innovación desarrolla un papel fundamental en todos los niveles educativos, y en el terreno universitario está íntimamente relacionada con la enseñanza virtual, siendo una de las formas básicas para su realización la incorporación de sistemas de información, herramientas de comunicación y elementos multimedia.

La mayoría de centros de educación superior están inmersos en la actualidad en el desarrollo del componente no presencial de una buena parte de sus materias mediante el establecimiento de campus virtuales, de hecho su desarrollo es una de las líneas de actuación del programa eLearning establecido por la Unión Europea para la integración efectiva de las nuevas tecnologías en los sistemas de educación y formación durante el periodo 2004-2006 (http://ec.europa.eu/education/ programmes/elearning/doc/dec es.pdf).

Para Teare et al (2002) el componente no presencial permite ganar flexibilidad e innovar en el campo del aprendizaje. Los entornos virtuales, como señalan los autores Fuentes et al (2004), suponen un reto en la sociedad actual por el impacto que han producido y su implantación en multitud de ámbitos, siendo la educación uno de los más importantes. Proporcionan un soporte fundamental para adecuar la metodología docente a las necesidades de los alumnos, ofreciendo claras ventajas, pero requiere una serie de cambios en el alumnado, profesores e instituciones. 
David Carabantes, Amparo Carrasco y Joaquim Alves

LA INNOVACIÓN A TRAVÉS DE ENTORNOS VIRTUALES DE ENSEÑANZA Y APRENDIZAJE

Se encuentran disponibles en el mercado distintos sistemas para el establecimiento de estos entornos virtuales de formación, con diferencias en cuanto al grado de sofisticación, pero que comparten una metodología de uso muy parecida, e incluso una apariencia similar. Incorporan por regla general utilidades para la creación y gestión de contenidos, junto con mecanismos de comunicación de forma síncrona (como el chat) y asíncrona como el correo electrónico y los foros de debate. Un mismo sistema suele presentar versiones para el mundo empresarial y para el universitario, entre los más extendidos se encuentran: WebCT (http:// www.webct.com), Blackboard (http://www.blackboard.com/), Edustance (http:// www.edustance.com/) y Oracle iLearning (http://ilearning.oracle.com/ilearn/en/ learner/jsp/login.jsp); de software libre destacan Moodle (http://moodle.org/) y Sakai (http://sakaiproject.org/).

Existen además distintos programas que mejoran las posibilidades que integran las plataformas educativas, de forma que por ejemplo el software Hot Potatoes (http://web.uvic.ca/hrd/hotpot/) permite desarrollar actividades atractivas para los alumnos como son los crucigramas. Respondus (http://www.respondus.com/), es otra herramienta que posibilita la creación en este caso de ejercicios en un formato estándar (IMS) compatible con diversas plataformas, de manera que tras generarse las preguntas y los criterios de realización se incluyen automáticamente para que el alumno los realice.

La implantación de las nuevas tecnologías en el marco educativo es uno de los pilares fundamentales en este marco europeo, por lo que es necesario realizar una evaluación de los programas existentes y aprender a gestionar el cambio que se produce, además, como afirma Vélaz (2003), en el nuevo marco de educación que se configura, los materiales formativos en el componente virtual de la educación deben de tener una serie de propiedades con el fin de que sean realmente didácticos. Para cumplir estos propósitos existen iniciativas desde los Vicerrectorados encargados en cada Universidad que permiten proporcionar apoyo económico para adaptarse al nuevo marco educativo.

Las posibilidades de la tecnología aplicada a la educación en los estudios universitarios permiten complementar la docencia tradicional a través de la Red, nunca sustituirla. Se configura así un medio de aprendizaje más dinámico, que de manera constante detecta las dificultades y las solventa de forma rápida y eficaz, permitiendo el mejor aprovechamiento de los recursos disponibles.

En este nuevo sistema, desaparecen los modelos convencionales de aprendizaje acumulativo y se buscan métodos pedagógicos centrados en el alumno, teniendo que 
David Carabantes, Amparo Carrasco y JOAQuim Alves

LA INNOVACIÓN A TRAVÉS DE ENTORNOS VIRTUALES DE ENSEÑANZA Y APRENDIZAJE

desempeñar el profesor unos roles a los que en ocasiones no estaba acostumbrado: desarrollando habilidades con las tecnologías de la información y la comunicación y poniendo en práctica actividades de aprendizaje individualizado, tareas que favorezcan el trabajo colaborativo y una metodología de aprendizaje basado en problemas.

Las tecnologías educativas adquieren un valor fundamental como apoyo a la docencia presencial: mayor calidad accesible a todo tipo de alumnos y capaz de ofrecernos formación en cualquier momento de nuestras vidas. Facilitan el proceso de convergencia hacia este nuevo marco, ya que permiten desarrollar sistemas de información que posibilitan el acceso a gran cantidad de contenidos, y las múltiples herramientas de comunicación favorecen la interacción bidireccional entre los integrantes del proceso. Se debe desarrollar una formación enfocada hacia las enseñanzas específicas en todos los campos científicos, junto con el desarrollo de las competencias transversales.

Desde las universidades europeas se ha aceptado el reto que suponen los objetivos de convergencia europea, y están adoptando (o ya están en marcha), las principales líneas de educación que permitirán la integración en este nuevo modelo educativo. Los estudios superiores ante la Europa del siglo XXI y la nueva cultura educativa apuestan por la integración del sistema universitario al nuevo espacio de enseñanza. Sus desafíos ante esta situación y las perspectivas de futuro en la era digital, estudian los problemas actuales de la universidad y recogen propuestas para adaptarla a las nuevas necesidades, la problemática de la universidad española y sus propuestas para acercarla al futuro, se enfocan principalmente en la integración de las nuevas tecnologías en este nuevo marco europeo.

Las experiencias que se describen a continuación proceden de las Escuelas Universitarias de Estudios Empresariales y de Enfermería, Fisioterapia y Podología de la Universidad Complutense de Madrid, y pretenden ejemplificar el uso de entornos virtuales para desarrollar actuaciones de innovación educativa en el ámbito de la docencia. Estos centros presentan una particularidad y es que al ser Diplomaturas las carreras que se imparten, este grado académico no se recoge por nuevo modelo europeo, lo que condiciona una clara transformación.

Se pretende de esta forma mejorar la realidad existente y responder a una clara necesidad educativa, ya que el nuevo modelo de educación exige el conocimiento y uso de las tecnologías de la información y comunicación en los estudios. Implica además una realidad de tipo sociocultural, ya que la sociedad demanda profesionales 
cada vez mejor formados en nuevas tecnologías para su perfecta integración en el mercado laboral, por ejemplo, el comercio electrónico en el campo de los estudios empresariales o las aplicaciones telemáticas en ciencias de salud.

\section{MATERIALES Y MÉTODOS}

La Escuela Universitaria de Estudios Empresariales (EUEE), de forma conjunta con la Facultad de Ciencias Económicas y Empresariales de la Universidad Complutense, y con la participación de profesores de análisis económico de otras universidades, inició durante el curso académico 2003-2004 un intento de mejorar el rendimiento del alumno en el proceso de enseñanza y aprendizaje a través de las nuevas tecnologías.

Mediante la concesión de tres Proyectos de Innovación Educativa (PIE): "microint" (Microeconomía Intermedia), "epolitic" (Economía Política) y "macroint" (Macroeconomía Intermedia), se implementaron los materiales didácticos de los profesores implicados en versiones electrónicas consultables vía web y en CD-ROM (Carrasco et al, 2004b).

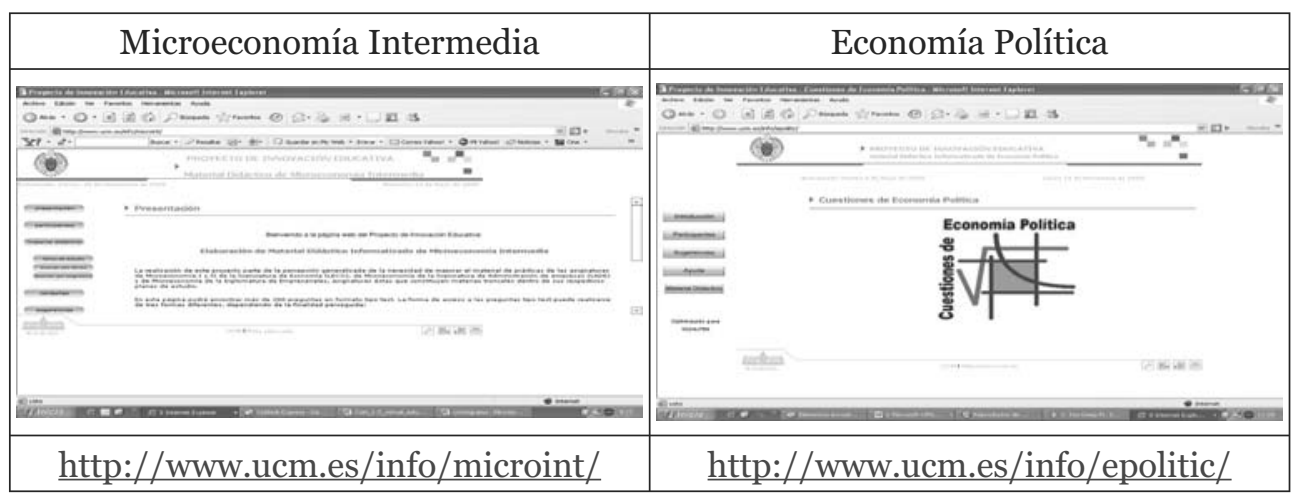

Se desarrollaron además dos experiencias de docencia presencial con apoyo virtual: Microeconomía (asignatura obligatoria de segundo curso) y Economía Política (asignatura troncal de primer curso), intentando mejorar la calidad de la actividad docente (Carrasco, 2004a). Para ello se ha utilizado la herramienta WebCT, a través de la cual se está realizando desde 2003 el Campus Virtual de la Universidad (https://www.ucm.es/info/uatd/CVUCM/index.php). 
En los espacios creados se incluyeron los apartados de presentación, contenidos, comunicación, agenda, actividades y herramientas de evaluación. Los materiales didácticos del curso se dispusieron de manera eficiente, flexible y rápida, creando módulos de contenidos que han sido muy apreciados por los alumnos, y superan a los apuntes tradicionales o a otros tipos de materiales pedagógicos. Las herramientas de comunicación que se incorporaron generaron flujos de información bidireccional eficientes en el acto formativo.

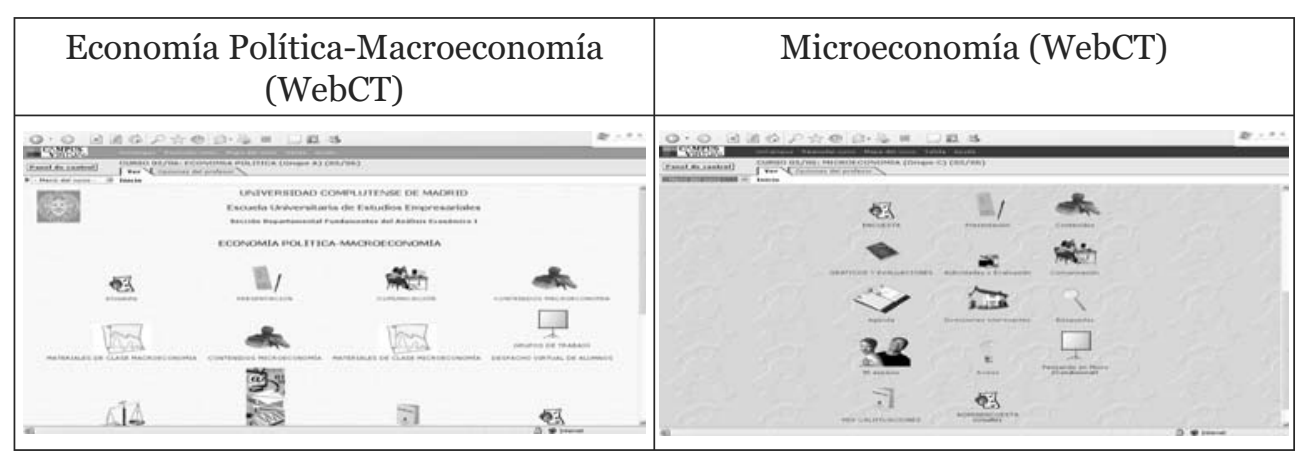

Durante el curso académico 2005-2006 se han implantado en el centro grupos piloto para la adaptación a la nueva metodología docente inherente a la implementación del Espacio Europeo de Educación Superior (EEES). Enfocado anteriormente a asignaturas optativas o de libre configuración, en este caso se ha apostado por una asignatura troncal y obligatoria de primer curso (sólo este centro y la Facultad de Matemáticas han optado por implementar un grupo piloto de estas características), siendo en Economía Política un número total de 23 alumnos.

En esta ocasión se ha elegido a un profesor sin docencia en el grupo piloto (el Subdirector de Calidad del centro) como interlocutor con los estudiantes para mejorar la comunicación por esta vía, y se han diseñado reuniones mensuales entre los profesores participantes en la experiencia para intercambiar impresiones y realizar un seguimiento común de los alumnos, lo que ha permitido el replanteamiento de algunas estrategias iniciales, como el mecanismo de constitución de los grupos y su volumen de participantes.

Como se ha indicado anteriormente, la asignatura de Economía Política se incorporó al Campus Virtual de la Universidad desde su creación, pero se ha replanteado su diseño con vistas a la nueva metodología docente de este grupo piloto. 
David Carabantes, Amparo Carrasco y Joaquim Alves

LA INNOVACIÓN A TRAVÉS DE ENTORNOS VIRTUALES DE ENSEÑANZA Y APRENDIZAJE

El objetivo principal es centrar más en el trabajo del alumno y se ha procedido a la elaboración de material de apoyo informatizado, tanto para la exposición de los temas por parte del profesor como para su utilización en el propio proceso de aprendizaje de los estudiantes. Se ha tratado de elaborar una ficha docente fundamentada en el enfoque de objetivos y competencias, conectando estrechamente los contenidos a los objetivos marcados, con un plan de trabajo realista para la consecución de éstos.

En cuanto a los materiales elaborados específicamente para el curso, se ha intentado superar en cierta medida el esquema más tradicional en nuestra disciplina: "resumen teórico-problemas resueltos-autoevaluación", utilizado en las experiencias anteriores que se habían plasmado en los Proyectos de Innovación Educativa referenciados anteriormente, y tres libros de ejercicios publicados por prestigiosas editoriales.

Se pretendía detectar algunos de los problemas que los alumnos de Empresariales encuentran a la hora de afrontar las asignaturas de Teoría Económica y de asimilar correctamente los conceptos estudiados, resultando tres las principales dificultades de los alumnos de Empresariales en su aproximación a las enseñanzas de Teoría Económica: su complejidad para relacionar los conceptos (que a menudo estudian de forma lineal), su falta de destreza para realizar análisis gráfico, y su práctica ausencia de motivación a la hora de estudiar conceptos y modelos abstractos, que aprecian generalmente como carentes de utilidad o demasiado alejados de la realidad y del desempeño de su actividad profesional.

Para el diseño de materiales que ayudarán a los alumnos a superar estas dificultades o deficiencias, se construyeron diferentes mapas conceptuales (según la metodología de Novak) en soporte informático con el propósito de ayudar a los estudiantes a comprender y asimilar conceptos básicos sobre Teoría Económica. Estos mapas conceptuales actuarían a modo de esquemas interrelacionados que suponen una forma diferente de presentar y organizar el conocimiento. Además, esta herramienta puede ser utilizada de forma autónoma por parte de los alumnos para organizar su proceso de aprendizaje, intercambiar información, estimular el espíritu crítico y aprender trabajando en grupo.

Respecto a la falta de competencia del alumnado en la construcción, utilización e interpretación de gráficos, fundamentales en esta disciplina, se han puesto a su disposición representaciones animadas sobre diferentes aspectos clave de la asignatura, con el fin de dotarlos de herramientas de aprendizaje autónomo, fácilmente utilizables, cuyo uso puede ser iterativo y su atractiva interfaz puede 
constituir un estímulo para el entrenamiento en la utilización de instrumentos que en cierta medida les resultan hostiles. Finalmente, se ha procedido a realizar una recopilación de diferentes aplicaciones prácticas de los conceptos y modelos teóricos objeto de estudio, que sean de utilidad para que los alumnos consideren la Teoría Económica como algo más próximo y relevante.

También se han incluido presentaciones en formato electrónico para las sesiones presenciales, problemas resueltos y ejercicios de autoevaluación, diseñando actividades como simulaciones en el aula, elaboración de trabajos en grupo, búsqueda dirigida de información y tutorías personalizadas, entre otras.

En el caso de la enseñanza en ciencias de la salud, Mihai y Navarro (2005) señalan que es en la actualidad cuando se ha comenzado a generalizar la utilización de Internet y aplicaciones multimedia. Desde los estudios de ciencias de la salud que se imparten en Escuela Universitaria de Enfermería, Fisioterapia y Podología (EUEFP), se ha apostado por la inclusión de entornos virtuales para conseguir un modelo mixto de aprendizaje "blended learning" en el que se combine de forma adecuada la docencia tradicional con el componente on-line. La disposición de WebCT para el Campus Virtual permitió que experiencias que se realizaban anteriormente a través de la página web del centro (o de otros entornos) pasaran a desarrollarse a través de este nuevo escenario de aprendizaje (Carabantes et al, 2004a).

Para asignaturas de pregrado como "Drogodependencias", impartidas en el segundo curso de Fisioterapia y Enfermería, se utilizó el modelo de las Actividades Académicas Dirigidas (AAD), consistente en la realización de hasta un 30\% del volumen total de créditos de la asignatura de forma no presencial a través del Campus Virtual, reduciendo el tiempo de permanencia en las aulas, fomentando la participación activa del alumnado y mejorando la interacción con el profesorado (Carabantes et al, 2004b).

Se ha ampliado el número de asignaturas participantes como "Teoría General de la Enfermedad" para los alumnos de primero de Podología, en la que por medio de la disposición de contenidos o de ejercicios se mejora el aprendizaje sobre las bases generales de las alteraciones que aparecen en el organismo humano cuando se instaura un proceso patológico. 
David Carabantes, Amparo Carrasco y Joaquim Alves

LA INNOVACIÓN A TRAVÉS DE ENTORNOS VIRTUALES DE ENSEÑANZA Y APRENDIZAJE

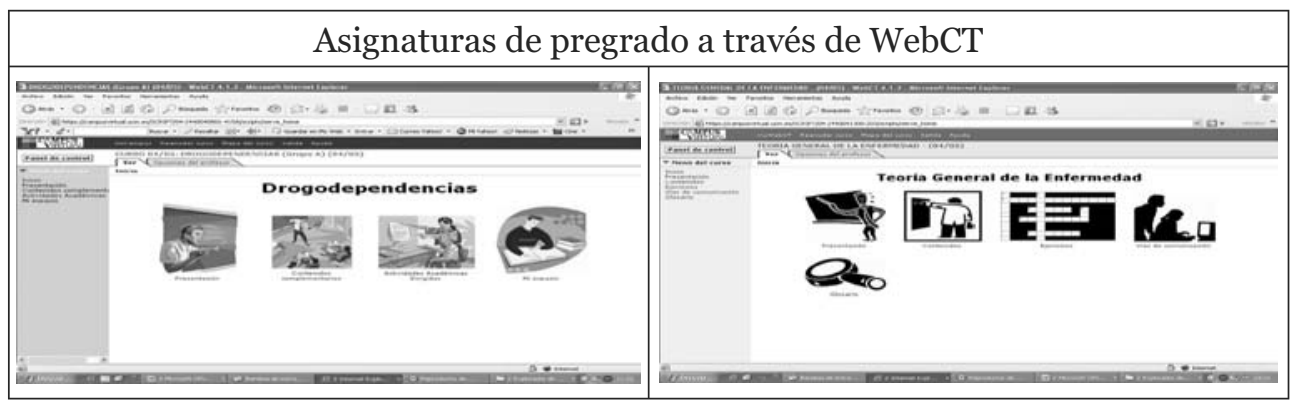

También se desarrollaron espacios virtuales en nuevos cursos de especialización como el Experto en Drogodependencias o en Fisioterapia Neurológica, títulos propios de la Universidad Complutense, para ampliar de esta forma el campo de actuación.

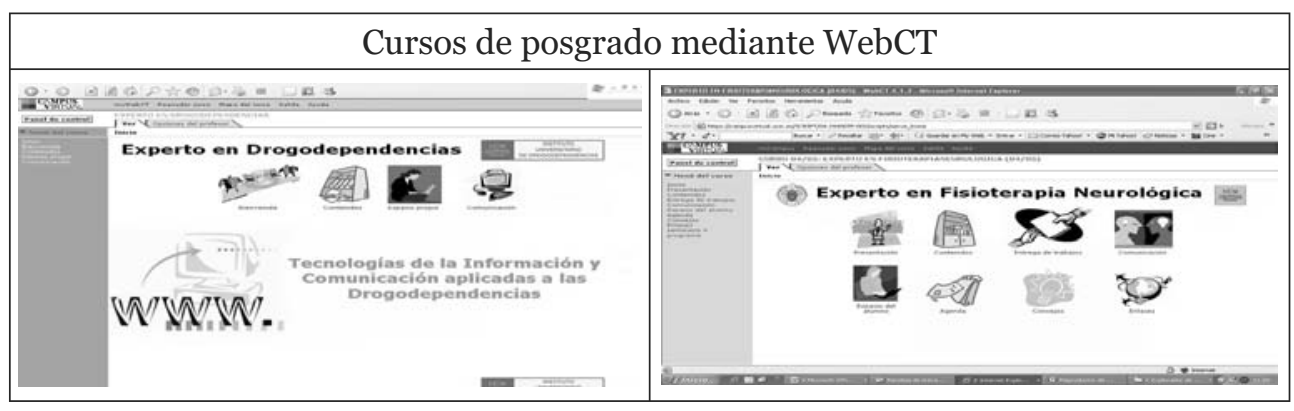

En el segundo cuatrimestre del curso académico 2005 se llevó a cabo una asignatura principalmente a través de Internet mediante la combinación de dos entornos virtuales: el sistema institucional del Campus Virtual y la plataforma InterCampus (Carabantes, 2005b), un espacio de trabajo este último ofrecido de forma gratuita por la Fundación Telefónica con la Universidad dentro del marco del espacio Intercampus de CampusRed (http://www.campusred.net/straining/cursos/ CURdcarabano2 CopiaEvaluacion2005/index2.asp).

La asignatura en cuestión se denominaba "Introducción a la Documentación y Búsqueda Bibliográfica”, una materia de libre configuración ofertada para todo el campus universitario, de un elevado número de estudiantes (300 matriculados) procedentes de varias disciplinas. Se pretendía iniciar al alumno desde el ámbito universitario en el proceso de documentación necesaria para la realización de trabajos 
de investigación, por lo que se explican las utilidades de las nuevas tecnologías aplicadas en la búsqueda bibliográfica para que posteriormente puedan desarrollar habilidades en su práctica profesional (Carabantes et al, 2005a).

La planificación de esta asignatura se basó en una serie de clases presenciales en la que se establecían las bases fundamentales y se resolvían dudas, utilizando las plataformas educativas para realizar una serie de actividades programadas. Se incluyó la participación de otros integrantes adicionales como personal de la biblioteca en calidad de profesores ayudantes que colaboraron en los temas sobre documentación, y los técnicos de las aulas de informática que ayudaron a los alumnos en la resolución de problemas al respecto.

En el diseño de los espacios se utilizó principalmente la configuración de WebCT por la posibilidad de entrega de las actividades para ser calificadas por el profesor y que así el alumno conociera los resultados con rapidez. La versatilidad y las herramientas que incorpora Intercampus, como el acceso a contenidos enciclopédicos o un buscador inteligente para búsquedas en Internet, permitió adaptarse a las necesidades de la asignatura.

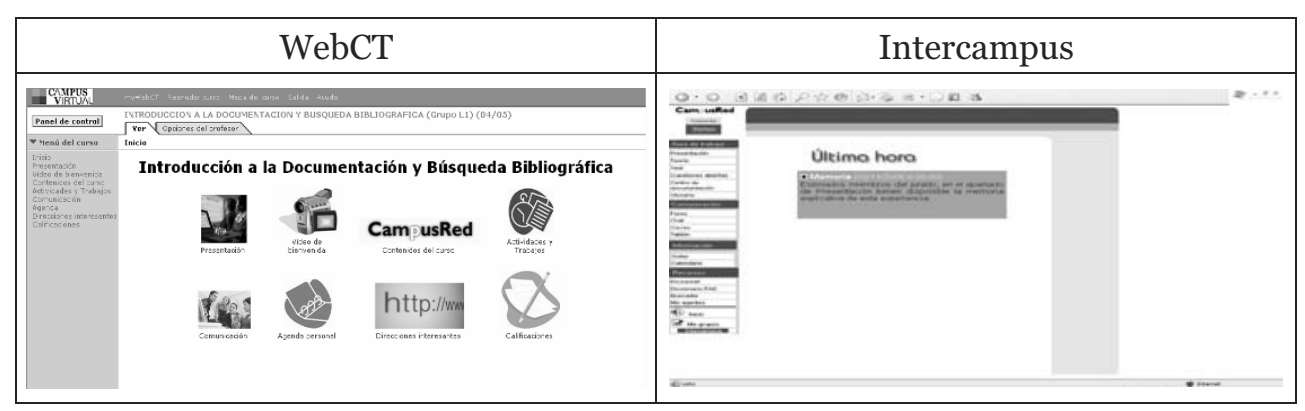

Las actuaciones conjuntas desarrolladas por los dos centros educativos en el curso 2005-2006, y las que se planifican en el futuro, van encaminadas a aumentar la participación de profesorado, incrementando las asignaturas con apoyo de componente virtual, y a mejorar los cursos diseñados anteriormente.

Desde ambos centros se han utilizado distintas herramientas complementarias al Campus Virtual como el programa Hot Potatoes, con el que se han desarrollado actividades que resultarán atractivas al alumno. 
David Carabantes, Amparo Carrasco y Joaquim Alves

LA INNOVACIÓN A TRAVÉS DE ENTORNOS VIRTUALES DE ENSEÑANZA Y APRENDIZAJE

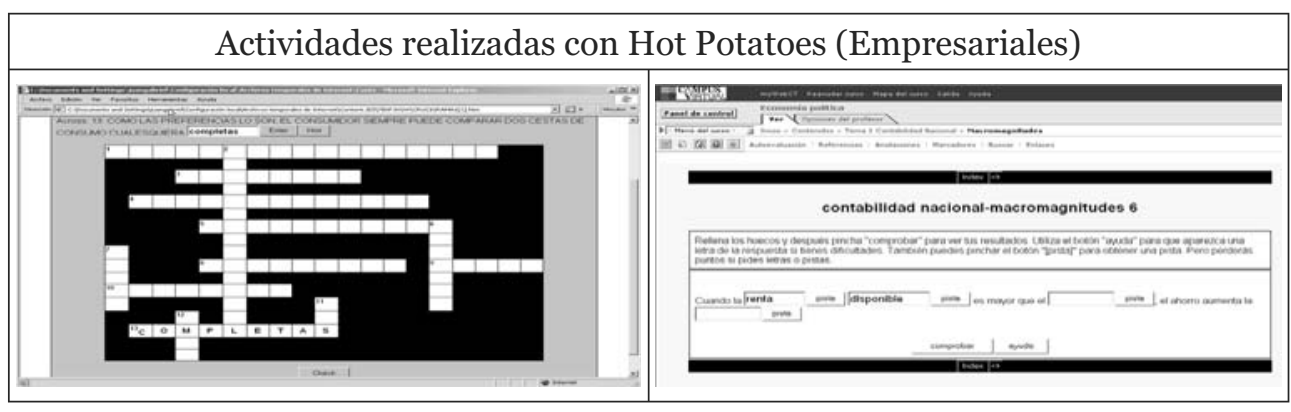

La creación de crucigramas electrónicos, ejercicios de completar espacios incluyendo pistas, relacionar conceptos o de ordenar términos, entendidas estas actividades como evaluación o como autoevaluación, son elementos que captan la atención del alumnado y motivan su participación. Es indudable que supone al profesor un trabajo adicional a la hora de establecer ejercicios de este tipo, pero por regla general tienen una muy buena aceptación por parte del estudiante, que en ocasiones espera a ver la creatividad del docente en este sentido.

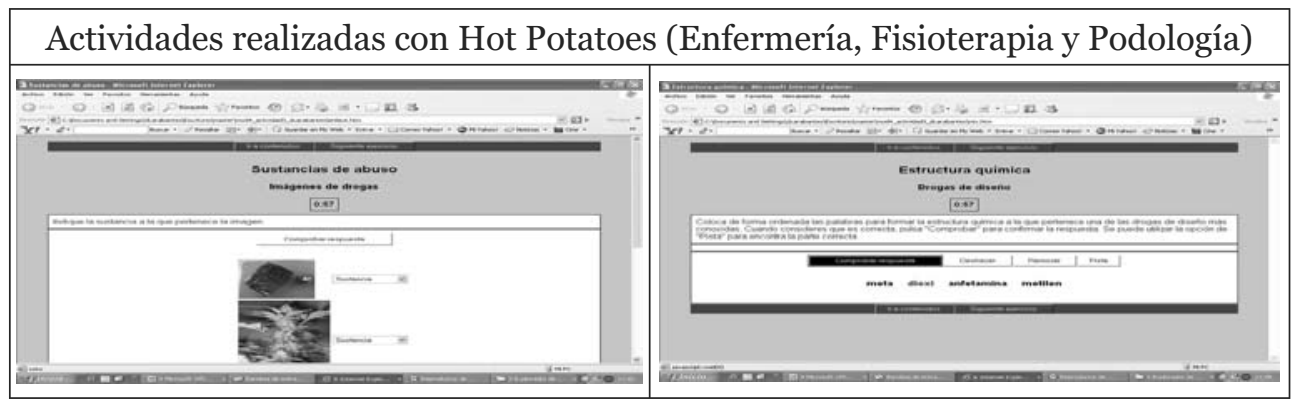

Además de intentar aumentar la calidad en la docencia, se ha potenciado desde estos centros la utilización de entornos virtuales para integrar la investigación en este nuevo modelo y poder coordinar grupos de trabajo, aspectos primordiales en el ámbito universitario.

La investigación es uno de los pilares fundamentales en el nuevo Espacio Europeo de Educación Superior. Los entornos virtuales se presentan como una herramienta fundamental para el uso de la tecnología educativa y potenciar la colaboración en el proceso científico entre profesor de distintas disciplinas, diferentes universidades 
y distancia geográfica, pero también en la investigación que pueden realizar conjuntamente profesor y alumno.

Los centros de educación superior tienen por lo tanto una doble misión en cuanto a la educación y a la investigación, se pretende despertar el espíritu crítico al alumnado para así motivar a la investigación, pero sin embargo, en algunos planes de estudio no existen por el momento contenidos de esta materia, sobre todo en enseñanzas de Escuelas Universitarias, en las que las asignaturas dedicadas al método científico y de la bioestadística han sido relegadas, y aunque con la esperanza de que en otras materias se trataran estos temas, no siempre ha sido así.

El desarrollo de la investigación es uno de los aspectos que permiten potenciar una disciplina, y en el momento actual se pueden utilizar las nuevas tecnologías para fomentar este proceso desde distintos ámbitos. A nivel de la educación universitaria existen propuestas, tanto en el grado como en cursos de especialización, que pretenden potenciar el aprendizaje del método científico a través de las tecnologías de la información y la comunicación.

En la EUEFP se han desarrollado experiencias de investigación en el pregrado y el posgrado, porque se pretendía potenciar la participación de los universitarios en este proceso para generar conocimiento. Antes de que el alumno termine sus estudios se encamina su aprendizaje hacia la investigación, con un curso voluntario sobre metodología básica y encauzando en este sentido a los estudiantes beneficiarios de una beca de excelencia. En los cursos de posgrado se intentaba fomentar la investigación para que los ya profesionales mejoren la situación de la investigación (Beneit et al, 2005).

Para el curso voluntario de investigación a nivel básico, se desarrolló a través del sistema Intercampus, un espacio de trabajo colaborativo para los estudiantes de las diferentes disciplinas, con el objetivo de que el alumno se familiarice con el método científico y los aspectos relacionados con las fases principales. La utilización de una metodología docente con apoyo en los sistemas informáticos, permite facilitar el aprendizaje sobre investigación y dinamizar su puesta en práctica para que así resulte más atractiva (Carabantes, 2005b).

En el pregrado, existen asignaturas específicas sobre metodología básica de la investigación que pretenden fomentar el espíritu científico en el alumno, usando las nuevas tecnologías para coordinar y ayudar en el desarrollo de trabajos tutorizados que pueden luego presentar en eventos específicos para estudiantes, como el Congreso 
David Carabantes, Amparo Carrasco y JOAQuim Alves

LA INNOVACIÓN A TRAVÉS DE ENTORNOS VIRTUALES DE ENSEÑANZA Y APRENDIZAJE

Europeo de Investigación en Pregrado de Ciencias de la Salud en la Comunidad de Madrid que se realiza anualmente en el Hospital Central de la Defensa, o las primeras Jornadas Complutenses de Investigación de Alumnos de Pregrado en Ciencias de la Salud que en esta edición se han realizado en la Facultad de Medicina (https:// campusvirtual.ucm.es/eventos/1ips.html)

Algunos de estos eventos se organizan mediante entornos virtuales, y entre sus participantes se encuentran los beneficiarios de las Becas de Excelencia, convocadas anualmente por la Dirección General de Universidades e Investigación a través de la Consejería de Educación de la Comunidad de Madrid dentro de la propuesta del Espacio Madrileño de Educación Superior, consistente en que los alumnos con buen expediente académico colaboren en tareas de investigación y docencia. Se consigue implicar así al alumnado de forma temprana en el campo científico y estimularlos para que encaminen su trayectoria en este sentido a través de los Programas Oficiales de Posgrado.

Se creó un seminario de trabajo en el Campus Virtual específico para los alumnos con beca de Aprovechamiento Académico Excelente (AAE), una ayuda que se convoca de forma anual por la Dirección General de Universidades e Investigación a través de la Consejería de Educación de la Comunidad de Madrid (http://www.emes.es/), y que está destinada a aquellos alumnos que posean una elevada calificación en su expediente académico al iniciar la carrera universitaria o cuando ya se estén cursando estudios superiores.

Las experiencias anteriores se realizaron durante el curso académico 2004-2005, y en el siguiente está proyectada también su realización. Se pretende conseguir la mayor difusión de los resultados de investigación que hacen los alumnos ayudados de profesores, de forma que algunas de las propuestas realizadas por el alumnado se presentaron y presentarán a congresos con esa filosofía.

Para potenciar la investigación en los cursos de posgrado y títulos propios, donde coexisten tanto alumnos que acaban de concluir recientemente sus estudios como aquellos que llevan una amplia trayectoria profesional, se han incluido materias relativas a la investigación, de forma que en el Experto en Fisioterapia Neurológica referenciado anteriormente, durante los cursos 2004-2005 y 2005-2006, uno de los primeros módulos de contenidos establece las bases de metodología de la investigación y de búsqueda bibliográfica para fundamentar el estado de la cuestión de un tema. 
El Master de Investigación en Cuidados para Enfermería, que ha puesto en marcha la Escuela Universitaria de Enfermería, Fisioterapia y Podología de la Universidad Complutense de Madrid, permite la posterior realización de estudios correspondientes al grado de doctor, y es por eso que incluye un módulo obligatorio en primer año implementado en WebCT sobre tecnologías de la información y comunicación.

Desde la EUEE se han realizado propuestas de desarrollo de trabajos de investigación junto con profesores de distintas universidades españolas y extranjeras, por ejemplo sobre buenas prácticas en la didáctica de economía en Escuelas de Ciencias Empresariales

La creación de un espacio en el Campus Virtual de Empresariales mediante las posibilidades de WebCT permitió la incorporación de las herramientas básicas para formar un grupo de trabajo en el que se pudieron intercambiar información, ficheros y sucesivas versiones del trabajo, herramientas de comunicación para establecer una comunicación fluida, permitió compartir ideas de forma fértil y dinámica, una agenda en la que se estableció un cronograma de trabajo. Se obtuvieron resultados tan buenos que el sistema permanece activo para futuras actuaciones.

Dada la importancia del establecimiento de redes de docencia e investigación con otros centros educativos en Europa, desde la EUEE se estableció a través del Campus Virtual UCM la coordinación de un grupo de trabajo para la consecución de un convenio internacional de doble titulación firmado por la Universidad Complutense de Madrid, País Vasco, Alicante, Burdeos y el Instituto Politécnico de Setúbal, firmado en el Ministerio de Educación en febrero de 2005 (Carrasco, 2005).

El espacio creado de esta manera mediante WebCT incluye las herramientas que se consideraron necesarias: contenidos, grupos de trabajo, foros, correo y agenda. Ha sido puesto a disposición de todos los participantes y ha servido como soporte del convenio, siendo un pilar fundamental en la aprobación del programa por parte del Ministerio de Educación Francés como título superior oficial. Además, esta siendo utilizado como instrumento para las tareas de coordinación entre los responsables del convenio, labores que pueden resultar muy complejas ya que intervienen profesores de distintos países, con diferentes idiomas, agendas sobrecargadas y hábitos culturales y académicos diversos.

La tarea de coordinación se ha visto facilitada enormemente por la utilización de este sistema, la experiencia ha permitido potenciar el trabajo cooperativo y los resultados atestiguan que ha sido muy alto el grado de satisfacción de los participantes. 
La apuesta de la EUEFP de utilización de los entornos virtuales para la gestión va encaminada a la coordinación de los Programas Oficiales de Posgrado, regulados en enero de 2005 y que van a cambiar el planteamiento del centro, enfocando estos estudios hacia el aprendizaje mixto en el centro ya que como máximo un $25 \%$ de las clases pueden ser presenciales.

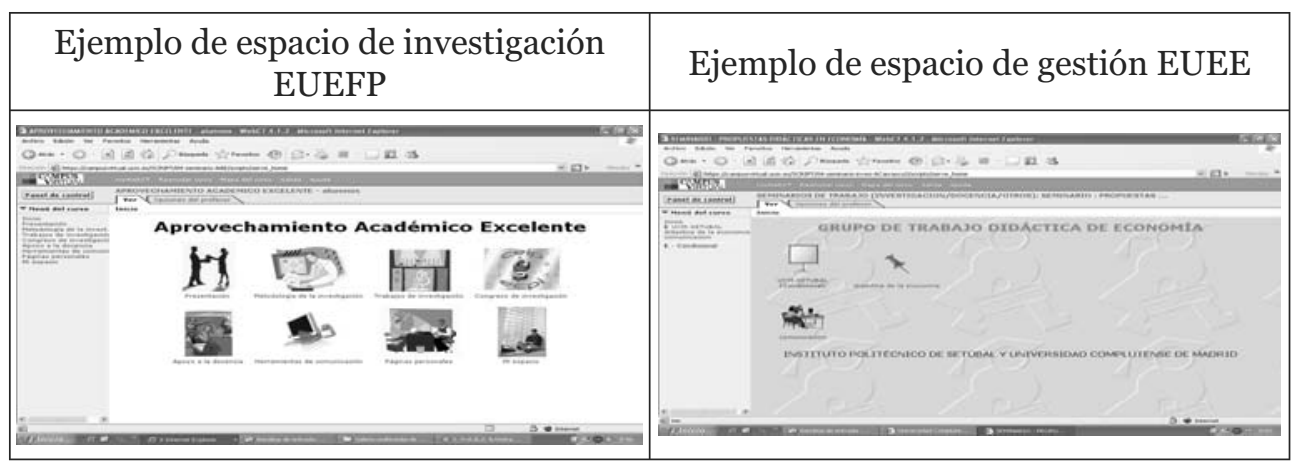

\section{RESULTADOS}

La Unidad de Apoyo Técnico y Docente al Campus Virtual (UATD-CV) ha venido desarrollando desde la implantación de este sistema, una valoración de la satisfacción de los alumnos mediante encuestas. Durante el curso 2005-2006 se ha implementado una modalidad de realización en la que el profesor pueda incluir en los espacios web de sus asignaturas unos cuestionarios, como el desarrollado para conocer las implicaciones del uso del Campus Virtual por parte del alumno, obteniendo los resultados de forma rápida y sencilla.

En nuestro caso, se han seleccionado un número representativo de encuestas realizadas por el alumnado en asignaturas tan dispares entre sí como Drogodependencias para el Grupo B de Enfermería, y Microeconomía para los alumnos de Empresariales. Se eligió el mismo número en cada caso (las primeras 65 encuestas) realizadas durante el segundo cuatrimestre del curso académico 20052006. Se quería comprobar, por un lado, si existían grandes diferencias entre los centros y profesorado, y seguidamente realizar la valoración global mediante la suma de todas las encuestas. 
A continuación se muestran los resultados de algunas de las preguntas que incluye el cuestionario y que ilustran la utilidad de los entornos innovadores para el desarrollo de innovación educativa, no obteniendo diferencias considerables entre los distintos centros y profesores.

En primer lugar, con respecto al grado de satisfacción que muestran los alumnos de la utilización de una plataforma de enseñanza basada en la web para su aprendizaje, más de la mitad de los encuestados consideran que están bastante satisfechos en este sentido, siendo muy alto el número de personas que se encuentran con un elevado grado de satisfacción (Gráfico 1).

El trabajo en el Campus Virtual con una herramienta de enseñanza virtual ha servido bastante al alumno para mejorar, afianzar o familiarizarse con la utilización de aplicaciones web e Internet. Además, se puede observar que un volumen considerable de los encuestados consideraban que el uso de esta herramienta ha sido de gran utilidad para este fin (Gráfico 2).

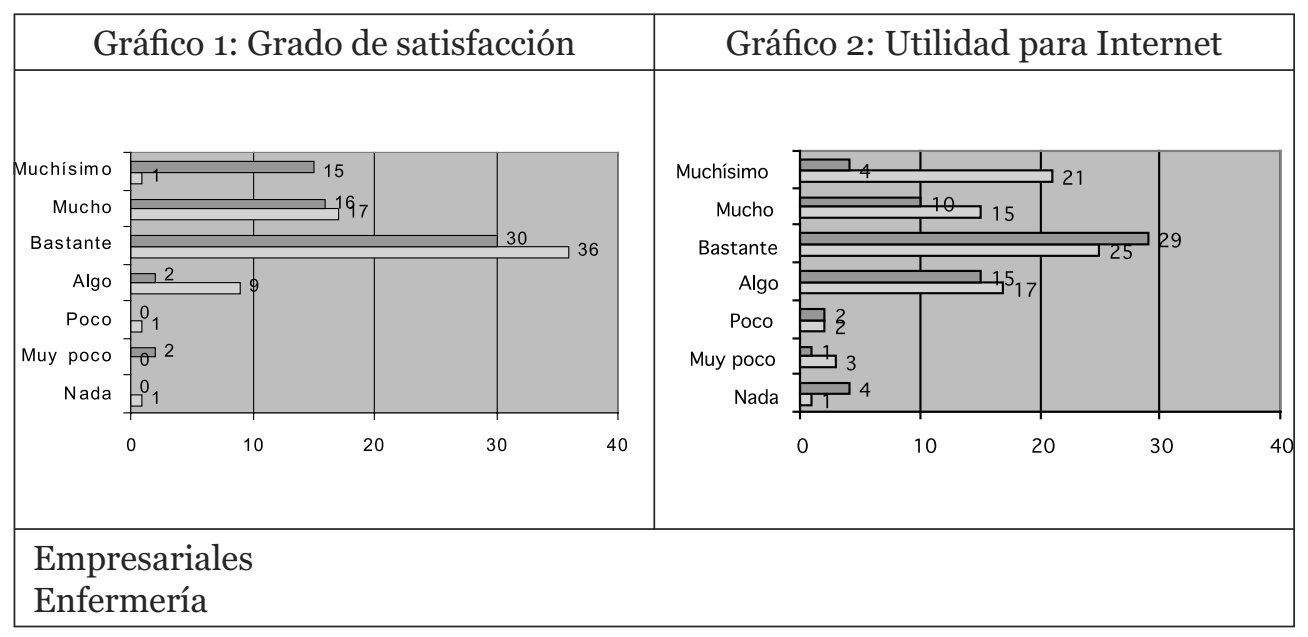

Disponer del Campus Virtual ha facilitado mucho la preparación de la asignatura por parte de los alumnos; en pocas ocasiones no ha influido (Gráfico 3). La valoración que hacen del grado de dificultad que tiene utilizar el Campus Virtual es nulo o muy poco (Gráfico 4). 


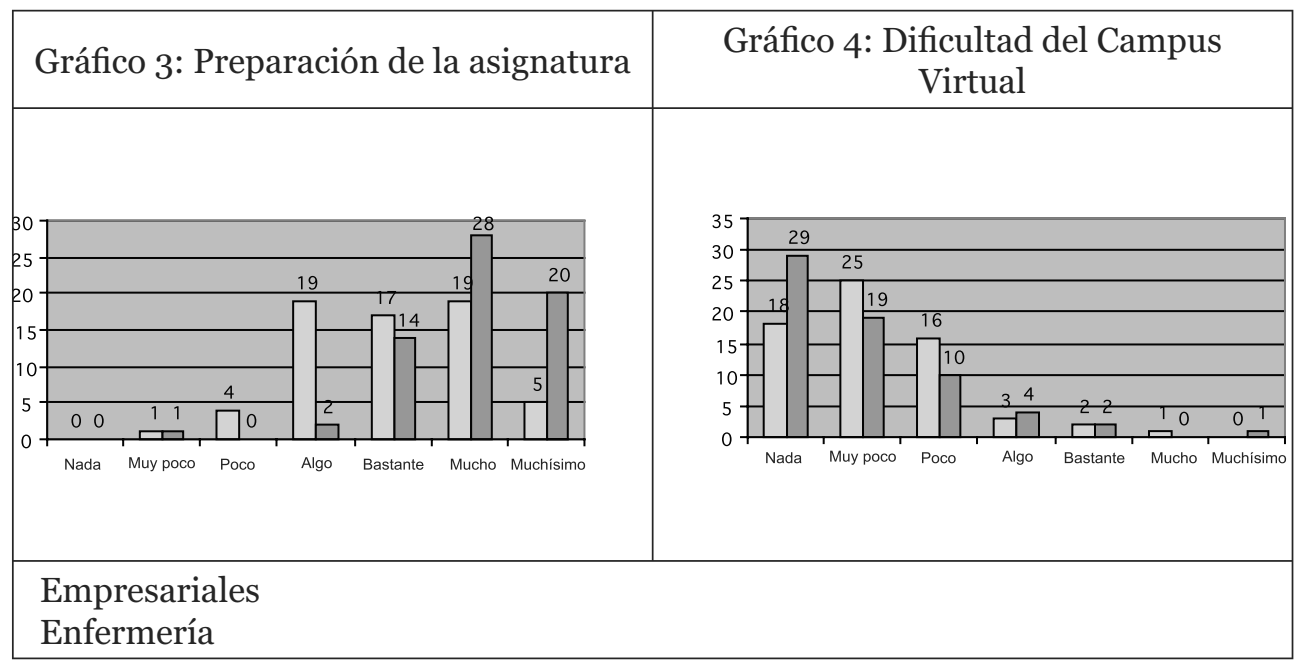

Prácticamente la mitad del alumnado valora su actividad en el Campus Virtual como satisfactoria (Gráfico 5), mientras que la actividad del profesorado en el Campus Virtual es valorada por el alumno como satisfactoria y altamente satisfactoria (Gráfico 6).

\begin{tabular}{|l|l|}
\hline Gráfico 5: Actitud del alumno & Gráfico 6: Actitud del profesor \\
\hline \multicolumn{3}{|c|}{ Actitud del alumno } & \\
\hline & \\
\hline
\end{tabular}


David Carabantes, Amparo Carrasco y JoAQuim Alves

LA INNOVACIÓN A TRAVÉS DE ENTORNOS VIRTUALES DE ENSEÑANZA Y APRENDIZAJE

\section{CONCLUSIONES}

Las experiencias presentadas demuestran las ventajas del uso de entornos virtuales en el establecimiento y desarrollo a través de la Red de actividades de aprendizaje. Los sistemas pedagógicos individualizados, el desarrollo de tareas y actividades que favorezcan el trabajo colaborativo, posibilitan un método eficaz en la transmisión de conocimientos y en la adquisición de habilidades por parte del alumno.

La implantación de sistemas de enseñanza mediante Internet ha permitido que los estudiantes dispongan de una mayor autonomía e implicación en su proceso de aprendizaje, con un incremento de la flexibilidad y accesibilidad. Las encuestas realizadas han reflejado que los alumnos, una vez vencidas sus reticencias iniciales han sido conscientes de los beneficios de su utilización y el atractivo de esta metodología.

Este modelo ha permitido que el alumnado dispusiera de una mayor autonomía, con un incremento de la flexibilidad y accesibilidad. Ha podido disfrutar de una variante metodológica sin las restricciones de tiempo y espacio, pudiendo aprender a buen ritmo, sin requerir una excesiva dedicación ni que resultara una carga. El estudiante ha autogestionado su tiempo y profundizado en su aprendizaje según sus necesidades.

Se ha mejorado la comunicación e interacción entre alumno y profesor, ya que a través de esta vía se vence la barrera de la timidez, favoreciendo la interacción y la vinculación entre los propios estudiantes, con un mayor contacto entre los integrantes y realizando un trabajo colaborativo efectivo. Se ha producido una participación más activa y efectiva del alumnado, estableciéndose un contacto que en ocasiones no se puede conseguir mediante las clases presenciales.

Las experiencias desarrolladas en este estudio muestran la evolución, los cambios necesarios, y las ventajas obtenidas en la integración de dos centros educativos en el nuevo modelo educativo, realizando una aproximación al modelo de aprendizaje que combine la docencia presencial con un componente virtual que permita aproximarse a la realidad sociocultural.

En definitiva, la integración de la docencia e investigación a través de la Red se ha hecho de forma efectiva, a lo que se han sumado actuaciones de gestión y coordinación que de otra manera hubiera sido imposible realizar, y que han conseguido como resultado final unos estudios universitarios cada vez más competentes, abiertos y de calidad. 


\section{REFERENCIAS BIBLIOGRÁFICAS}

Beneit, J. V.; Martín, P.; Atín, M. A.; Álvarez, M. C.; Pacheco, E.; Carabantes, D. (2005). Entornos virtuales para el aprendizaje y desarrollo de investigación en pregrado y posgrado, en Fernández-Valmayor, C. y otros II Jornada Campus Virtual UCM: Cómo integrar investigación y docencia. Madrid: Editorial Complutense, 310-318.

Carabantes, D.; Sanz, F.; Álvarez, M. C.; García, C.; Beneit, J. V. (2005). Las plataformas educativas en la docencia de la documentación para desarrollar investigación, en Fernández-Valmayor, C. y otros II Jornada Campus Virtual UCM: Cómo integrar investigación y docencia. Madrid: Editorial Complutense, 319-322.

Carabantes, D. (2005). El trabajo colaborativo en un entorno virtual. [en línea]. Disponible en: http://www.campusred. net/congreso/pdfscomunicaciones/ com dcaraban cong intercampus.pdf [consulta 2005, 30 de mayo].

Carabantes, D.; Pacheco, E.; García, C.; San Juan, A.; Beneit, J. V. (2004).Utilidad y uso de las nuevas tecnologías de la información y comunicación en el modelo de enseñanza y aprendizaje de la Escuela Universitaria de Enfermería, Fisioterapia y Podología, en Fernández-Valmayor, C. y otros I Jornada Campus Virtual UCM: En apoyo del aprendizaje en la Universidad, hacia el espacio europeo de educación superior. Madrid: Editorial Complutense, 189-195.

Carabantes, D.; Pacheco, E.; García, C.; Beneit, J. V. (2004). Aplicación de las nuevas tecnologías de información y comunicación en el modelo europeo de Educación Superior: La experiencia de la Escuela Universitaria de Enfermería, Fisioterapia y Podología de la Universidad Complutense de Madrid. [en línea]
Disponible en: http://www.virtualeduca. org/2004/es/actas/2/1.2.1.doc [consulta 2005, 30 de mayo].

Carbonell, J. (2002). El profesorado y la innovación educativa, en Cañal, P. La innovación educativa. Madrid: Akal, 1126.

Carrasco, A. (2005). El Campus Virtual más allá de la docencia: dos experiencias de utilización del Campus Virtual en otras actividades académicas, en FernándezValmayor, C. y otros II Jornada Campus Virtual UCM: Cómo integrar investigación y docencia. Madrid: Editorial Complutense, 323-326.

Carrasco, A. (2004). Incorporación al Campus Virtual UCM de dos asignaturas de Teoría Económica en Fernández-Valmayor, C. y otros I Jornada Campus Virtual UCM: En apoyo del aprendizaje en la Universidad, hacia el espacio europeo de educación superior. Madrid: Editorial Complutense, 23-29.

Carrasco, A.; Gracia, E.; Iglesia, C. de la. (2004). Líneas didácticas para una nueva estrategia en la Enseñanza Superior de Teoría Económica: dos experiencias docentes de incorporación de TIC, en Fernández-Valmayor, C. y otros IJornada Campus Virtual UCM: En apoyo del aprendizaje en la Universidad, hacia el espacio europeo de educación superior. Madrid: Editorial Complutense, 196-201.

Cebrián, M. (2003). Enseñanza virtual para la innovación universitaria. Madrid: Narcea.

Fuentes, L.; Casilari, E.; Troya, J. M.; Sandoval, F. (2004). Entornos virtuales colaborativos. Málaga: Universidad de Málaga/Fundación Auna. 
Mihai, B.; Navarro, V. (2005). Comparación del aprendizaje en internet con la clase convencional en estudiantes de medicina, en Argentina. Educación Médica; 8(4), 204-207.

Rivas, M. (2000). Innovación educativa: teoría, procesos y estrategias. Madrid: Síntesis.
Teare, R.; Davies, D.; Sandelands, E. (2002). Organizaciones que aprenden $y$ formación virtual. Barcelona: Gedisa.

Vélaz de Medrano, C. (2003). La «mediación múltiple»: creación de un sistema de enseñanza/aprendizaje a distancia a partir del diseño de un plan general de recursos didácticos de la disciplina. Revista Iberoamericana de Educación a Distancia, 6 (2), 9-28.

\title{
PALABRAS CLAVE
}

Innovación educativa, Docencia, Investigación, Gestión, Entornos virtuales

\section{KEYWORDS}

Educational innovation, Teaching, Investigation, Management, Virtual environments

\section{PERFIL ACADÉMICO DE LOS AUTORES}

David Carabantes Alarcón es licenciado en Documentación y doctor por la Universidad Complutense de Madrid. Máster en nuevas tecnologías aplicadas a la educación por el Instituto Universitario de Posgrado. Sus principales líneas de investigación se centran en los entornos virtuales de enseñanza/aprendizaje y los sistemas de evaluación de la calidad en Internet. En la actualidad es profesor en la Escuela Universitaria de Enfermería, Fisioterapia y Podología de la Universidad Complutense de Madrid

\author{
Dirección Postal David Carabantes Alarcón \\ Escuela Universitaria de Enfermería, Fisioterapia y Podología \\ Universidad Complutense de Madrid \\ Avenida Complutense s/n 28040 Madrid - España \\ Correo electrónico: dcaraban@enf.ucm.es
}


David Carabantes, Amparo Carrasco y Joaquim Alves

LA INNOVACIÓN A TRAVÉS DE ENTORNOS VIRTUALES DE ENSEÑANZA Y APRENDIZAJE

Amparo Carrasco Pradas es licenciada en Económicas y profesora titular en el Departamento de Fundamentos del Análisis Económico I en la Universidad Complutense de Madrid desde 1990. En la actualidad desempeña su actividad docente e investigadora en la Escuela Universitaria de Estudios Empresariales, donde es coordinadora del Campus Virtual desde su creación, participando además en todas las iniciativas de adaptación al EEES. Entre sus principales líneas principales de investigación se encuentran las TIC y la Educación Superior, adaptación al EEES, innovación educativa y calidad docente.

Dirección Postal Amparo Carrasco Pradas

Escuela Universitaria de Estudios Empresariales

Universidad Complutense de Madrid Avda Islas Filipinas $n^{0} 3$

28003 Madrid - España

Correo electrónico: apradas@emp.ucm.es

Joaquim Daniel Alves Pais es Licenciado en Educación por el ISCE (Portugal), actualmente cursa estudios de Doctorado en Creatividad Aplicada en la Universidad Complutense de Madrid. Coordinador pedagógico de la empresa de Formación Profesional "Cubo Mágico" (Viseu-Portugal), viene participando como diseñador en diferentes Proyectos de Innovación Educativa en la UCM y es autor de diferentes trabajos en el área de Creatividad y Nuevas Tecnologías. Líneas de Investigación: Creatividad y Formación del profesorado, TIC y Educación Superior.

Dirección postal: $\quad$ Joaquim Daniel Alves Pais

C/ Ríos Rosas 4528003 Madrid

España

Correo electrónico: danalves@ya.com

Fecha de entrega: 23.10.06

Fecha de aceptación: 20.12.06 